Open Access

\title{
Thymoquinone enhances cisplatin-response through direct tumor effects in a syngeneic mouse model of ovarian cancer
}

\author{
Andrew J. Wilson ${ }^{1,4^{*}}$ D, Jeanette Saskowski ${ }^{1}$, Whitney Barham², Fiona Yull ${ }^{2,3}$ and Dineo Khabele ${ }^{1,3}$
}

\begin{abstract}
Background: Ovarian cancer is the most lethal gynecologic malignancy characterized by the frequent development of resistance to platinum chemotherapy. Finding new drug combinations to overcome platinum resistance is a key clinical challenge. Thymoquinone (TQ) is a component of black seed oil that exerts multiple anti-tumorigenic effects on cells, including inhibition of NF-KB and promotion of DNA damage. We aimed to determine whether TQ enhances cisplatin cytotoxicity in cultured ovarian cancer cells and in an established murine syngeneic model of ovarian cancer.
\end{abstract}

Methods: Ovarian cancer cell viability in vitro was measured by sulforhodamine B (SRB) assays, and drug interactions tested for synergism by isobologram analysis. ID8-NGL mouse ovarian cancer cells stably expressing an NF-kB reporter transgene were injected intra-peritoneally into C57BL/6 mice. After 30 day TQ and/or cisplatin treatment, we measured the following indices: tumor burden (ascites volume, number of peritoneal implants and mesenteric tumor mass); NF-KB reporter activity (luciferase assay); protein expression of the double-strand DNA break marker, pH2AX(ser139), the proliferation markers, Ki67/mib-1 and PCNA, and the apoptosis markers, cleaved caspase-3, cleaved PARP and Bax; and mRNA expression of NF-KB targets, TNF- $a$ and IL-1 $\beta$. Two-tailed Mann-Whitney tests were used for measuring differences between groups in mouse experiments.

Results: In SRB assays, TQ and cisplatin synergized in ID8-NGL cells. In mice, cisplatin significantly reduced cell proliferation and increased apoptosis in tumors, resulting in decreased overall tumor burden. Combining TQ with cisplatin further decreased these indices, indicating co-operative effects between the drugs. TQ treatment promoted cisplatin-induced pH2AX expression in cultured cells and in tumors. While NF-kB inhibition by TQ induced anti-tumor effects in vitro, we made the unexpected observation that TQ alone increased both tumor NF-kB activity and formation of ascites in vivo.

Conclusions: TQ enhanced cisplatin-mediated cytoxicity in ovarian cancer cells in vitro and in a mouse syngeneic model, effects associated with increased DNA damage. However, our results strongly caution that TQ treatment alone may have an overall deleterious effect in the immunocompetent host through stimulation of ascites. Since TQ is a potential candidate for future clinical trials in ovarian cancer patients, this finding has considerable potential relevance to the clinic.

Keywords: NF-kB activity, Ovarian cancer, Syngeneic mouse model, Macrophages, Thymoquinone, Cisplatin

\footnotetext{
* Correspondence: andrew.j.wilson@vanderbilt.edu

${ }^{1}$ Department of Obstetrics and Gynecology, Division of Gynecologic

Oncology, Vanderbilt University School of Medicine, Nashville, TN, USA

${ }^{4}$ Department of Obstetrics and Gynecology, Vanderbilt University Medical

Center, B1100 Medical Center North, Nashville, TN 37232, USA

Full list of author information is available at the end of the article
} 


\section{Background}

Ovarian cancer is the most common cause of death from gynecologic malignancies in the United States [1]. Most women with epithelial ovarian cancers are diagnosed with advanced, metastatic disease characterized by widespread peritoneal carcinomatosis and abdominal ascites [2]. The development of resistance to platinum chemotherapy (carboplatin and cisplatin) is common in advanced disease [3]. Therefore, identifying new drugs to improve platinum response is critical to prolonging the life of women with refractory disease.

Thymoquinone (TQ) is a product of the medicinal plant Nigella sativa which has promising anti-tumor efficacy in preclinical models of human cancer [4-7]. Multiple molecular mechanisms of action have been described for the demonstrated ability of TQ to reduce tumor growth and survival in these preclinical studies. These include activation of tumor suppressor genes such as PTEN and p21, reducing pro-inflammatory and angiogenic signals via inhibition of NF-kB signaling, an important molecular link between inflammation and cancer [8-13], and induction of DNA damage through generation of reactive oxygen species (ROS) [4-6]. Early clinical trials have shown promising lack of toxic effects in patients with symptoms of cardiovascular disease such as hypertension and hypercholesterolemia [6]. Only one Phase 1 trial has been reported for thymoquinone administration in 21 cancer patients, with no toxic or therapeutic effects detected over treatment times ranging from 1 to 20 weeks [14]. Definitive trials for establishing safe and effective doses of TQ in cancer patients are currently lacking, but are well supported by preclinical data [4-7].

Several mechanisms of resistance to platinum compounds in cancer cells have been identified [15]. First, cisplatin treatment is known to induce NF-kB [16], and NF- $\mathrm{kB}$ inhibitors potentiate the anti-tumor activity of various cytotoxic agents [17]. Second, cisplatin induces double-strand DNA breaks by intercalating into DNA [15], and its effects are reduced in ovarian cancer cells with intact DNA repair capacity [18]. We have shown previously that drugs which promote DNA damage or inhibit DNA repair (e.g. histone deacetylase inhibitors) can sensitize ovarian cancer cells to cisplatin and DNAdamaging drugs $[19,20]$. Since TQ has multiple cellular effects that could potentiate cisplatin response, we hypothesized that TQ would sensitize ovarian cancer cells cultured in vitro and in our syngeneic model to the cytotoxic effects of cisplatin.

Most preclinical models are limited by the fact that drug effects are tested on cancer cells in the absence of the supporting tumor microenvironment, essential for cancer progression in vivo. For this reason, we generated a mouse syngeneic model using ID8 mouse ovarian cancer cells grown intra-peritoneally in C57BL/6 mice [21]. The cells have a stably integrated NF- $\mathrm{kB}$ reporter plasmid, allowing for quantification of tumor NF- $\mathrm{kB}$ activity in response to drug treatment during intraperitoneal abdominal carcinomatosis accompanied by ascites formation.

In this study, we show that combined TQ and cisplatin treatment induced synergistic anti-tumor effects in cultured ID8-NGL cells, and reduced tumor burden, proliferative and apoptotic markers in ID8-NGL-derived tumors. These combinatorial effects were associated with enhanced expression of the DNA damage marker, $\mathrm{pH} 2 \mathrm{AX}(\operatorname{ser} 139)$, compared to either drug alone. Although TQ-mediated inhibition of NF-kB was observed in vitro, our syngeneic model showed an unexpected increase in tumor NF- $\mathrm{kB}$ activity and ascites volume with TQ treatment alone. These results emphasize the potential of targeting DNA damage as a therapeutic approach in ovarian cancer, but also that strongly caution TQ may have an overall deleterious effect through promotion of ascites formation. Since TQ is a likely candidate for future clinical trials in cancer patients [5-7], this finding has considerable potential relevance to the clinic.

\section{Materials and methods \\ Cell culture}

Mouse ovarian cancer cells stably expressing a NF- $\mathrm{kB}$ reporter plasmid, ID8-NGL [21], were cultured in $10 \%$ FBS-supplemented DMEM High-Glucose medium with $400 \mu \mathrm{g} / \mathrm{ml} \mathrm{G} 418$, and passaged by standard techniques. The human ovarian cancer cell lines, OVCAR3 and NCI/ADRRES were cultured as previously described [22]. Cultured ID8-NGL cells were treated with increasing concentrations of cisplatin (Sigma Chemical Co., Cat\# 479306) and/or the NF-кB inhibitor, thymoquinone (TQ; Sigma Chemical Co., Cat\# 274666).

\section{Cell viability assays}

Sulforhodamine B (SRB) assays were used to determine cell proliferation and cytotoxicity in response to TQ and/or cisplatin as previously described [22]. Absorbance was measured at $510 \mathrm{~nm}$ using a Spectramax M5 spectrophotometer (Molecular Devices, Sunnyvale, CA) in the High-Throughput Screening Core of the Vanderbilt Institute of Chemical Biology. The interaction between fixed ratios of TQ and cisplatin was measured with the Combination Index (CI) method [23]. A CI level of $<0.9$, $\mathrm{CI}=0.9-1.1$ and $\mathrm{CI}>1.1$ indicates synergy, additivity and antagonism respectively, between drug combinations.

\section{Animal model and drug treatment}

Wild-type C57BL/6 mice were injected intra-peritoneally (IP) with $1 \times 10^{7}$ ID8-NGL cells suspended in $200 \mu \mathrm{l}$ sterile PBS [21]. Thirty days after ID8-NGL injection, mice were randomized into the following treatment groups: vehicle (PBS), TQ (20 mg/kg thrice weekly), 
cisplatin ( $2 \mathrm{mg} / \mathrm{kg}$ weekly) and the TQ/cisplatin combination via the IP route for 30 days $(n=5)$. No signs of drug toxicity were observed in the single or combination treatment mice. Tumor progression was monitored by body weight and abdominal girth measurements. At time of sacrifice, abdominal ascites fluid was extracted with hypodermic syringe, and volume measured. Tumor implants in the peritoneal wall and the mesentery were measured, then harvested and snap frozen or formalin-fixed for further analysis. The experimental protocol was reviewed and approved by the Institutional Animal Care and Use Committee at Vanderbilt University.

\section{Luciferase assays}

Luciferase activity was measured in harvested tumors following tissue homogenization in $1 \mathrm{ml}$ reporter lysis buffer, and in whole cell protein extracts from cells grown in vitro, using the Promega Luciferase Assay system (Cat\#4030). Activity was analyzed using a GloMax Luminometer (Promega, Madison, WI). Results were expressed as relative light units (RLU) normalized for protein content, as measured by the Bradford assay (Bio-Rad, Cat\# 500-0002).

\section{RNA extraction and quantitative RT-PCR (QPCR)}

RNA from snap-frozen tumors was isolated using the RNeasy Mini kit (Qiagen, Valencia, CA) and QPCR performed as described [21]. Steady-state mRNA levels of the established NF- $\mathrm{B} B$ targets, TNF- $\alpha$ and IL-1 $\beta$, were expressed relative to corresponding GAPDH levels using the comparative $2^{\Delta \Delta C t}$ method [24]. Primers sequences used were as previously described $[21,25,26]$.

\section{Immunofluorescence}

Processing, embedding and sectioning of formalin-fixed ID8-NGL tumor tissue, and hematoxylin and eosin staining for histology, were performed in The Allergy/Pulmonary \& Critical Care Med Division Immunohistochemistry Core at Vanderbilt [27]. Immunofluorescence analysis of formalinfixed paraffin-embedded tumor tissue or methanol-fixed cultured ID8-NGL cells was performed using standard techniques [21, 19]. The following primary antibodies: mouse monoclonal anti-pH2AX(ser139) (EMD Millipore, Cat\# 05-636, 1:250 dilution); rabbit polyclonal antiKi67/Mib-1 (Abcam, Cat\# ab16667; 1:200 dilution); and rabbit polyclonal anti-cleaved caspase-3 (Cell Signaling Technology, Cat\# 9661; 1:100 dilution), were used. Secondary antibody used was goat anti-rabbit Alexa Fluor 488 (Life Technologies, Cat\# 11070) (all 1:200 dilution). Images were acquired and analyzed as previously described [21, 19]. For quantifying the percentage of, where applicable, tumor cells or macrophages positive for these proteins, at least 5 independent fields were assessed with at least 200 cells counted per sample.

\section{Western blotting}

In ID8-NGL cells treated with TQ $(25 \mu \mathrm{M})$ and/or cisplatin $(1 \mu \mathrm{M})$, or in drug-treated tumors, whole cell protein isolation, subcellular fractionation, western blotting and signal detection were performed as described [28, 29]. Primary antibodies used were rabbit polyclonal anti-PARP (Cell Signaling Technology; Cat\# 9542; 1:1000 dilution), mouse monoclonal anti-PCNA (Santa Cruz, Cat\# PC10, 1:1000 dilution), rabbit polyclonal anti-Bax (EMD Millipore, Cat\# 06-499, 1:1000 dilution), and mouse monoclonal anti-pH2AX(ser139) (EMD Millipore, Cat\# 05-636, 1:500 dilution). Mouse monoclonal anti- $\beta$-actin (Sigma Chemical Co., Cat\# A5441 1:10000 dilution) and rabbit polyclonal anti-H2AX (EMD Millipore, Cat\# 06-627, 1:500 dilution) were used as loading control, where appropriate.

\section{Statistical analysis}

Unless otherwise indicated, values shown for in vitro experiments were the mean $+\mathrm{SE}$ of 3 independent experiments, with comparison of groups performed by 2-tailed Student's $t$ test. Comparison of groups in in vivo experiments was performed by 2-tailed Mann-Whitney test. A $p$ value $<0.05$ is considered statistically significant.

\section{Results}

TQ enhances anti-tumor effects of cisplatin in cultured ovarian cancer cells

Here, we aimed to determine whether TQ can sensitize ovarian cancer cells cultured in vitro to the cytotoxic effect of cisplatin. First, the combination of TQ and cisplatin was tested in SRB cell growth and viability assays. In ID8-NGL mouse ovarian cancer cells. the drug combination induced synergistic inhibitory effects on cell viability, as shown by a Combination Index significantly less than 1 at Effective Doses (ED) ED50, ED75 and ED90 (Fig. 1a and b). Furthermore, the pro-apoptotic effects of cisplatin were markedly enhanced by combination TQ treatment (Fig. 1c), indicating that apoptosis was an important mechanism of action of the anti-tumor effects of the combined drugs.

The TQ and cisplatin combination was also tested in the established human ovarian cancer cell lines, OVCAR3 and NCI/ADR-RES, which are relatively resistant to cisplatin [22]. A synergistic decrease in viability of OVCAR3 and NCI/ADR-RES cells was observed with combination treatment with TQ and cisplatin (see Additional file 1: Figure S1).

TQ is known to have a wide range of cellular effects that may contribute to its anti-tumor actions, including inhibition of NF- $\mathrm{kB}[4,30]$. Cisplatin-induction of NF- $\mathrm{kB}$ is an established mechanism of cisplatin-resistance in cancer cells [16]. Therefore, we determined effects of combining TQ and cisplatin on NF- $\mathrm{KB}$ activity in ID8-NGL cells. As shown in Fig. 1d, TQ reduced NF-kB reporter activity by approximately $50 \%$ in luciferase assays after treatment for 

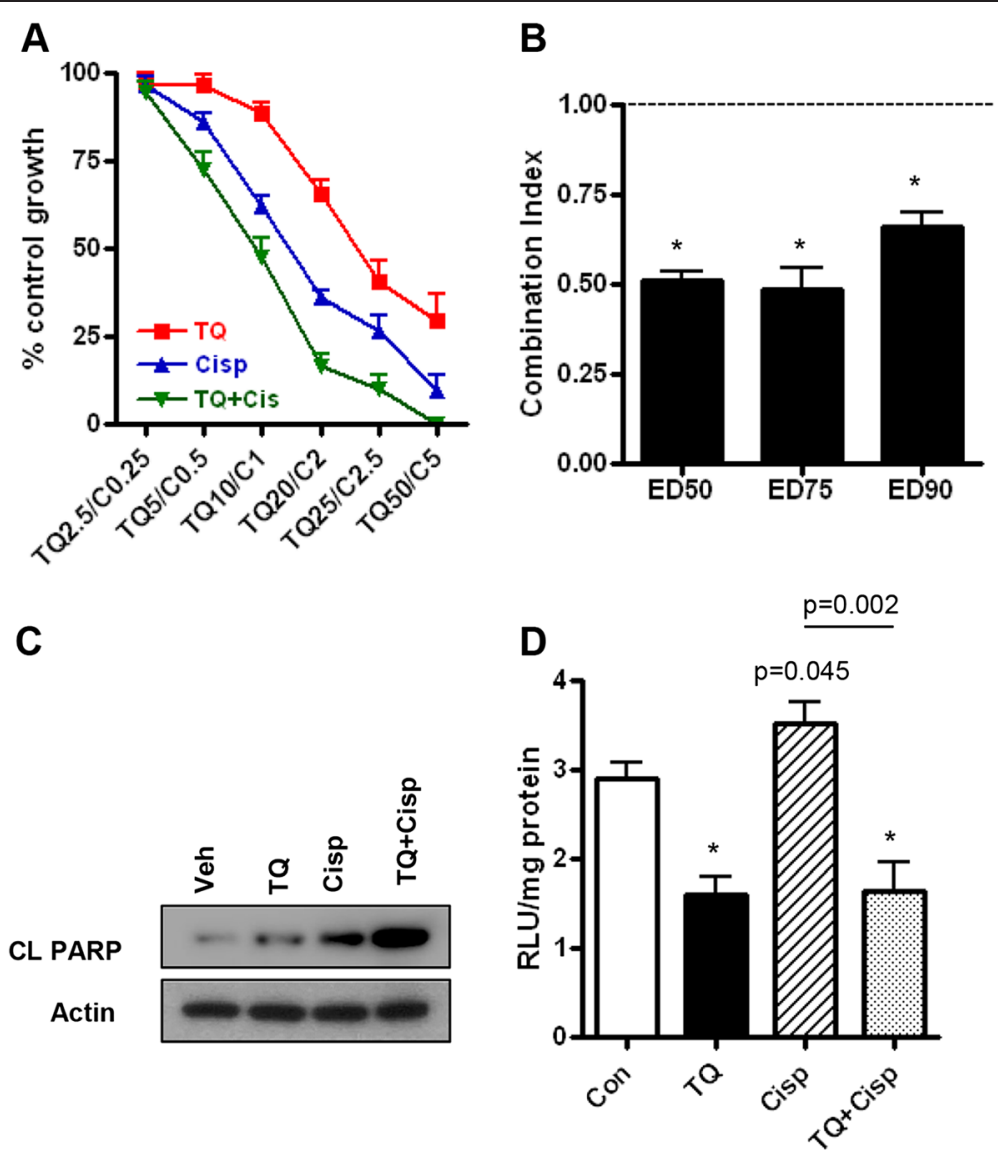

Fig. 1 TQ and cisplatin synergistically decrease cell viability in cultured ID8-NGL cells. a SRB assay showing effects of TQ and/or cisplatin (concentrations in $\mu \mathrm{M}, 72 \mathrm{~h}$ ) in the mouse ovarian cancer cell line, ID8-NGL, expressed as a percentage of control. b Isobologram analysis for ID8-NGL cells showing Combination Index for Effective Doses (ED) ED50, ED75 and ED90. A Combination Index < 1 indicates a synergistic drug interaction between TQ and cisplatin. Values are mean \pm SD for 3 experiments; ${ }^{*} p<0.01$ below 1 , Student's $t$ test. c Western blot analysis of cleaved PARP (CL PARP) following $24 \mathrm{~h}$ treatment of ID8-NGL cells with TQ $(25 \mu \mathrm{M})$, cisplatin $(1 \mu \mathrm{M})$ or the combination. Actin was used as a loading control. $\mathbf{d}$ Luciferase assay of ID8-NGL cells following $24 \mathrm{~h}$ treatment with vehicle, TQ $(25 \mu \mathrm{M})$, cisplatin $(1 \mu \mathrm{M})$ or the combination. Values are mean \pm SD for 3 experiments; ${ }^{*} p<0.01$ compared to control, Student's $t$ test

$24 \mathrm{~h}$. Cisplatin induced a modest increase in NF-kB reporter activity $(20.1 \pm 2.7 \%, p=0.045)$ (Fig. 1d), which was reduced to levels comparable to TQ alone by combining TQ and cisplatin.

TQ enhances anti-tumor effects of cisplatin in a syngeneic mouse model of ovarian cancer

Based on our in vitro results, we hypothesized that TQ treatment would produce co-operative effects when combined with cisplatin. We have shown in a previous study that TQ treatment for 10 days, starting at day 30 after tumor injection, reduced NF- $\mathrm{kB}$ activity as expected in ID8-NGL-derived tumors [21]. However, at this relatively early time point, tumor burden was minimal and was not readily quantifiable. Therefore, we chose to treat mice for 30 days to maximize our indices of tumor burden (ascites volume, number of peritoneal implants and mesenteric tumor mass) at the 60 day study end-point [21].
Treatment with TQ alone induced a 2-fold increase in ascites volume at day 60 compared to vehicle-treated mice (Fig. 2a). However, there was no overall difference in peritoneal (Fig. 2b) or mesenteric (Fig. 2c) tumors in mice treated with TQ only compared to vehicle. We confirmed these observations for TQ-only treatment in several additional experiments (data not shown). Cisplatin alone reduced all three indices of tumor burden by $>80 \%$ compared to vehicle-treated mice. Combining TQ and cisplatin resulted in enhanced reduction in peritoneal implants and mesenteric tumors compared to either drug alone, with a similar effect on ascites volume that just failed to reach statistical significance.

We next determined drug effects on cell proliferation and apoptosis in the tumors. Immunofluorescence analysis of tumors demonstrated that cisplatin reduced the percentage of cells positive for the proliferation marker, Ki67/mib-1 and increased cells showing expression of 

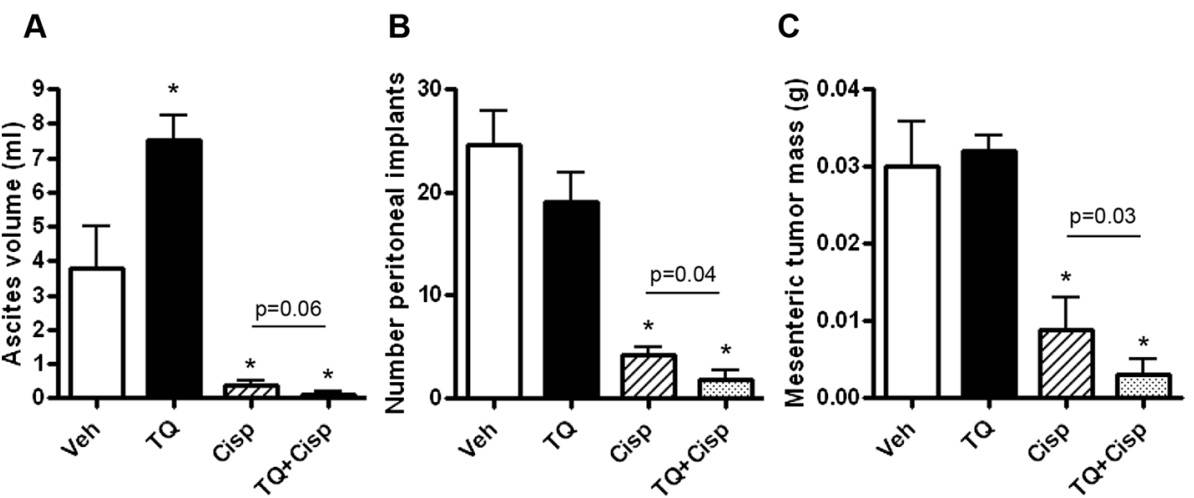

Fig. 2 TQ and cisplatin combine to decrease tumor burden. BL/6 mice were injected with ID8-NGL cells. Thirty days after tumor cell injection, mice were randomized and treated with PBS vehicle (Veh), TQ (20 mg/kg thrice weekly), cisplatin (Cisp; 2 mg/kg weekly) or the TQ/cisplatin combination for 30 days and sacrificed. At sacrifice, a ascites fluid volume, $\mathbf{b}$ number of peritoneal implants and $\mathbf{c}$ mesenteric tumor mass were measured. Values are mean + SD for 5 mice per group; ${ }^{*} p<0.01$ relative to vehicle; all $p$ values determined were by Mann-Whitney test

the apoptosis marker, cleaved caspase-3 (Fig. 3a and b), consistent with effects on tumor burden. Compared to cisplatin alone, the TQ/cisplatin combination group displayed significantly greater levels of apoptosis (Fig. 3a and b). We then independently validated our results by western blot analysis of two additional markers of apoptosis, cleaved PARP and Bax. As shown in Fig. 3c and d, both TQ and cisplatin significantly increased expression of cleaved PARP and Bax, consistent with their effects on cleaved caspase-3 expression. Moreover, combining the drugs significantly increased expression of cleaved PARP and Bax above that of cisplatin alone (Fig. 3c and d). Similarly, expression of PCNA was significantly reduced by TQ and cisplatin alone, and the TQ/cisplatin combination

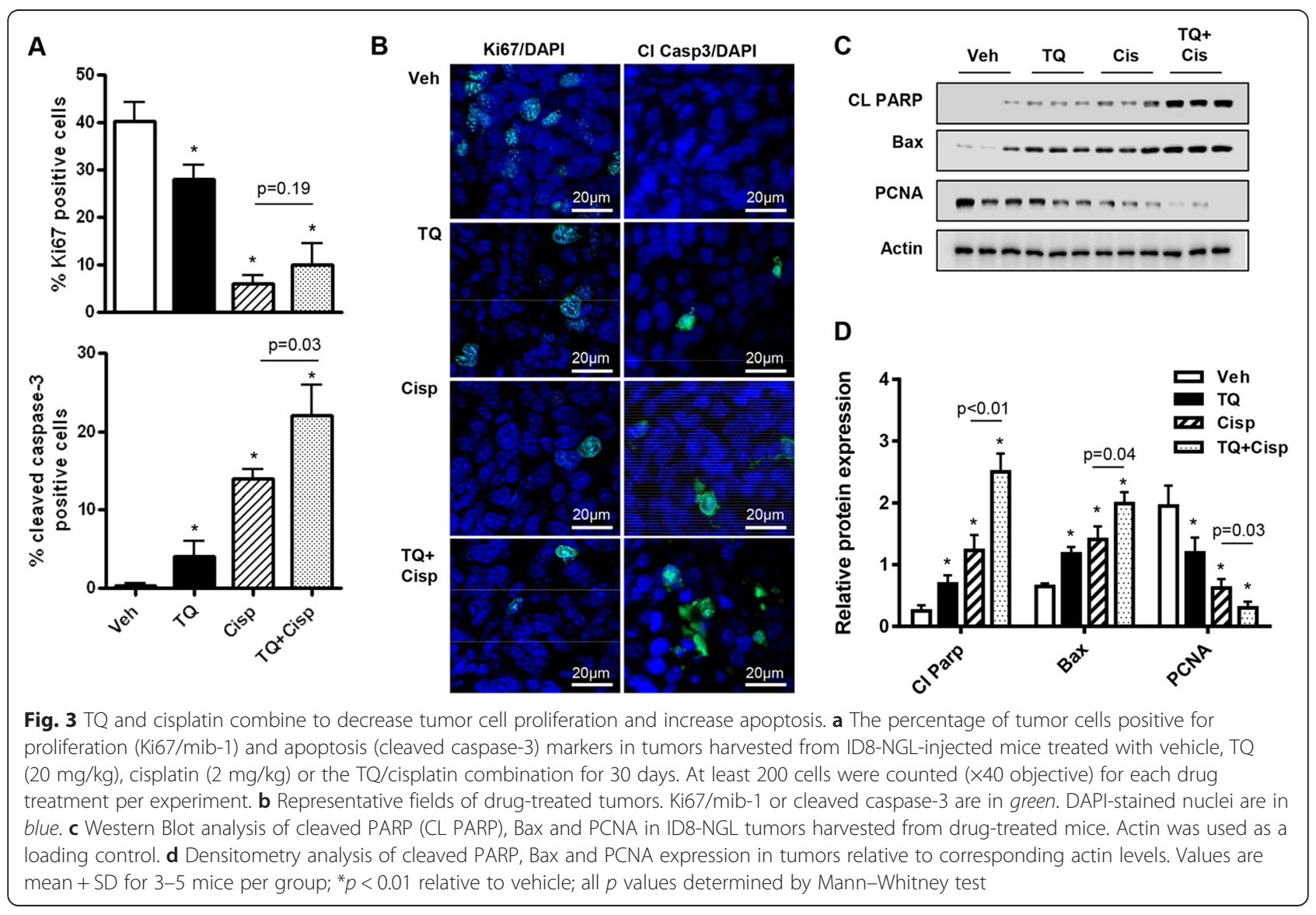


induced significantly greater effects than cisplatin alone (Fig. 3c and d). Therefore, we showed the anti-tumor effects of the TQ/cisplatin combination involved both enhanced cell apoptosis and reduced cell proliferation.

Contrasting drug effects on NF- $\mathrm{kB}$ reporter activity were observed in harvested ID8-NGL tumors compared to cultured cells. As shown in Fig. 4a, treatment with TQ for 30 days unexpectedly led to an overall increase in NF- $\mathrm{kB}$ reporter activity in luciferase assays. However, combining TQ and cisplatin abrogated TQ-stimulation, leading to overall similar levels compared to vehicle and cisplatin alone (Fig. 4a). Supporting our results, QPCR analysis of steady-state mRNA levels of the established NF- $\mathrm{kB}$ targets, TNF- $\alpha$ and IL- $1 \beta$, in drug-treated tumors revealed a similar pattern of effect to the luciferase assays (Fig. 4b).

\section{TQ enhances cisplatin-induced DNA damage in ovarian cancer cells}

Results from our in vivo experiments clearly indicate that TQ-mediated inhibition of NF-kB activity was not a mechanism underlying the co-operative effects of the $\mathrm{TQ} /$ cisplatin combination. Because of the pronounced increase in, particularly, apoptosis mediated by the combination in both cultured cells and in tumors, we explored alternative mechanisms underlying this pro-apoptotic effect. Since TQ is known to induce DNA damage in cancer cells $[31,32]$, and sustained DNA damage is linked to apoptosis [33], we hypothesized that TQ enhanced cisplatininduced DNA damage in cultured ID8-NGL cells and in ID8-NGL-derived tumors in vivo. We identified persistent pH2AX(ser139) activation, which is associated with DNA damage-induced apoptosis [33] and is a sensitive surrogate marker of cytotoxicity in ovarian cancer cells $[19,20]$.
In cultured ID8-NGL cells, we determined patterns of pH2AX(ser139) staining by immunofluorescence analysis following $24 \mathrm{~h}$ drug treatment $[19,20]$ (Fig. 5). Compared to vehicle-treated cells, TQ significantly decreased the number of $\mathrm{pH} 2 \mathrm{AX}(\operatorname{ser} 139)$-negative cells ( $<6$ foci), and increased the number of cells displaying both intermediate (6-20 foci) and high (>20 foci) pH2AX(ser139) expression. Cisplatin treatment alone had similar effects, with the exception that the proportion of cells displaying pan-nuclear staining (no discrete, countable foci) was also significantly increased compared to controls. Compared to either drug alone, combination treatment further reduced the number of $\mathrm{pH} 2 \mathrm{AX}$-negative cells, and the proportion of cells showing intermediate, high and pan-nuclear $\mathrm{pH} 2 \mathrm{AX}$ (ser139) expression.

We found a similar pattern of effect in drug-treated tumors, where the highest percentage of tumor cells positive for $\mathrm{pH} 2 \mathrm{AX}$ (ser139) in immunofluorescence assays was observed following TQ and cisplatin combination treatment, significantly higher than for cisplatin alone (Fig. 6a and b). We obtained similar results when $\mathrm{pH} 2 \mathrm{AX}$ (ser139) expression was measured by western blot (Fig. 6c and d).

\section{Discussion}

Platinum resistance is common in ovarian cancer, and identifying novel drug combinations to enhance efficacy of platinum drugs such as carboplatin and cisplatin is a promising strategy. In this study, we tested the ability of TQ to sensitize ovarian cancer cells to cisplatin treatment in established preclinical models. First, we demonstrated that the combination of TQ and cisplatin had synergistic inhibitory effects on cell viability and survival in cultured human and mouse ovarian cancer cells. Second, compared to either drug alone, there were enhanced effects of the combination
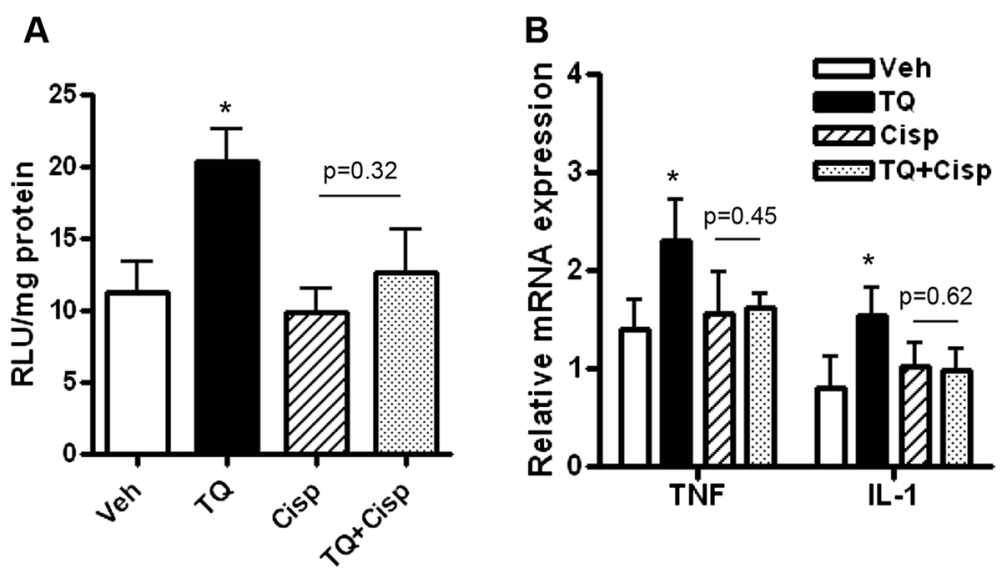

Fig. 4 NF-KB activity in ID8-NGL tumors is not inhibited by treatment with TQ and/or cisplatin. BL/6 mice were injected with ID8-NGL cells and treated with vehicle, TQ $(20 \mathrm{mg} / \mathrm{kg})$, cisplatin $(2 \mathrm{mg} / \mathrm{kg})$ or the combination for 30 days. a Luciferase assays of NF-kB reporter activity in harvested ID8-NGL tumors. $\mathbf{b}$ QPCR analysis of the established NF-KB target genes, TNF- $a$ and IL-1 $\beta$, in harvested tumors. Relative expression was calculated using corresponding GAPDH levels as the internal control. Values are mean + SD for 5 mice per group; ${ }^{*} p<0.01$ relative to vehicle; all $p$ values determined by Mann-Whitney test 


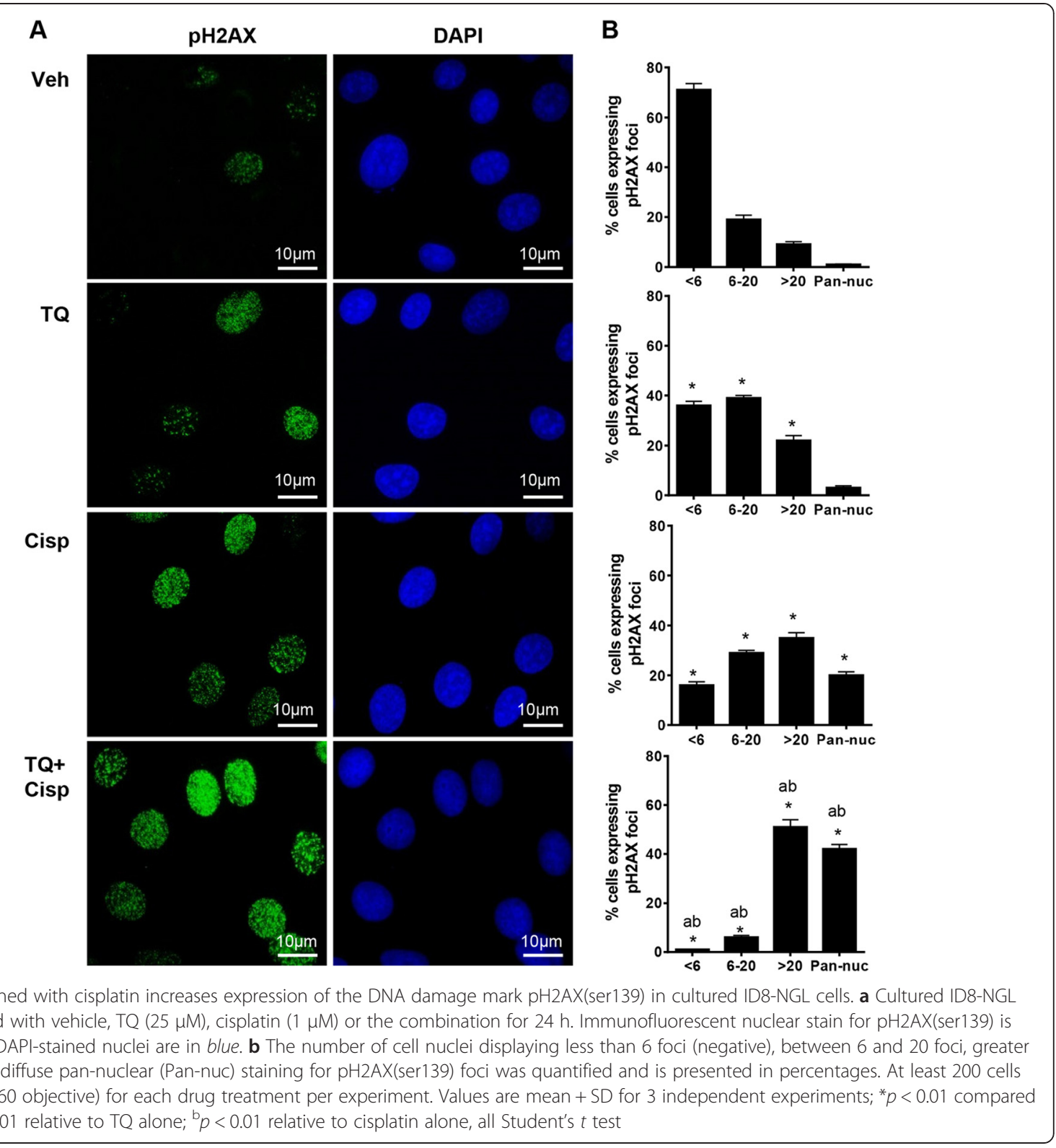

on established indices of tumor burden (peritoneal implants and mesenteric tumor mass), proliferation and apoptosis in a syngeneic mouse ovarian cancer model [21].

TQ is known to have a myriad of cellular effects in tumor cells, including promotion of DNA damage through generation of reactive oxygen species and inhibition of NF- $\kappa B$ activity [4-6]. Our results indicate that TQ was able to enhance DNA damage induced by cisplatin in both cultured cells and in tumors, consistent with previous studies from this laboratory showing that drugs which promote DNA damage sensitize ovarian cancer cells to the cisplatin-mediated cytotoxicity $[19,20]$. In contrast, TQ-mediated inhibition of NF- $\mathrm{B}$ activity could not explain the enhancement of the cisplatin response in cultured cells and tumors. First, cisplatin only weakly induced NF- $\mathrm{kB}$ activity in vitro, an established mechanism of cisplatin-resistance [16]. Second, in contrast to cultured cells in vitro, TQ induced tumor NF- $\mathrm{kB}$ reporter activity and mRNA levels of the key NF-kB targets, IL-1 $\beta$ and TNF- $\alpha$, in drug-treated mice.

We acknowledge that the tumor microenvironment could impact drug response in our syngeneic model. For example, it is likely that the unexpected increase in ascites formation and activity of the NGL NF- $\kappa B$ reporter in tumors following the 30-day TQ treatment was mediated through drug effects on the tumor microenvironment. We have previously shown that TQ treatment for 10 days effectively reduces NGL reporter activity within tumors in vivo [21]. Furthermore, thrice-weekly TQ treatment results in persistent inhibition of NF- $\mathrm{kB}$ activity in cultured 

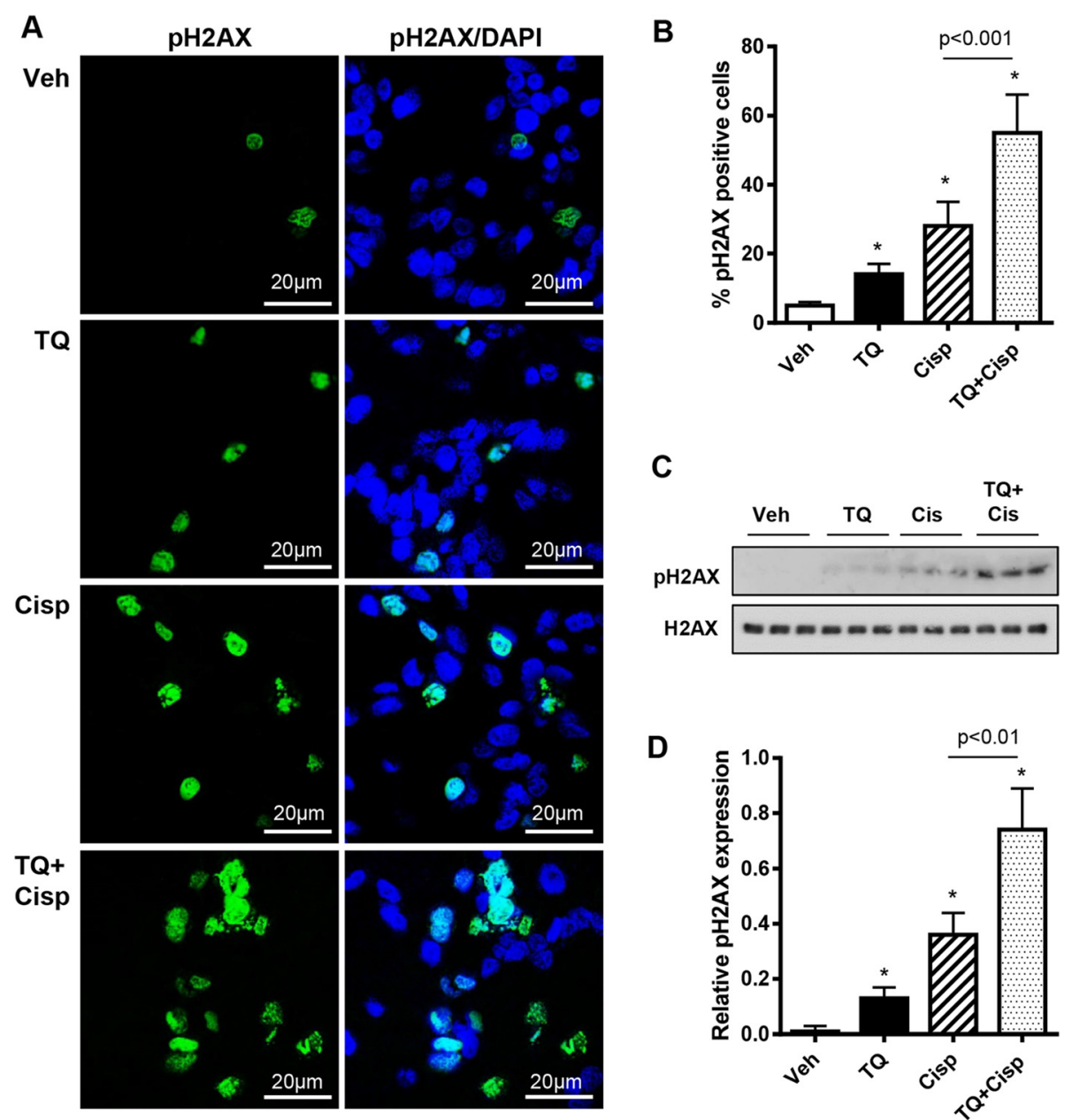

Fig. $6 \mathrm{TQ/cisplatin} \mathrm{combination} \mathrm{increases} \mathrm{pH2AX(ser139)} \mathrm{expression} \mathrm{in} \mathrm{ID8-NGL-derived} \mathrm{tumors.} \mathrm{a} \mathrm{Representative} \mathrm{fields} \mathrm{showing} \mathrm{pH2AX(ser139)}$ staining (green) and DAPI-stained nuclei (blue) in tumors harvested from ID8-NGL-injected mice treated with vehicle, TQ (20 mg/kg), cisplatin ( $2 \mathrm{mg} / \mathrm{kg}$ ) or the TQ/cisplatin combination for 30 days. b The percentage of tumor cells positive for $\mathrm{PH} 2 \mathrm{AX}(\mathrm{ser} 139)$ in drug-treated tumors. At least 200 cells were counted ( $\times 40$ objective) for each drug treatment. c Western Blot analysis of pH2AX(ser139) expression in tumors. Total H2AX was used as a loading control. $\mathbf{d}$ Densitometry analysis of pH2AX(ser139) expression in tumors relative to corresponding H2AX levels. Values are mean + SD for 3-5 mice per group; ${ }^{*} p<0.01$ relative to vehicle; all $p$ values determined by Mann-Whitney test

ID8-NGL cells in vitro for up to 21 days (unpublished observations). Identifying drug-induced alterations in specific inflammatory cell populations such as macrophages and dendritic cells, and possibly related mechanisms by which prolonged TQ exposure can induce NF-kB activity and ascites formation, will be the focus of future studies.

A possible microenvironmental role for TQ-induction of NF- $\mathrm{KB}$ activity and ascites following longer periods of treatment is supported by a recent study showing that the proteasome inhibitor, bortezomib, induces pro-inflammatory effects on the tumor microenvironment, particularly macrophages, subsequently promoting tumor progression in a mouse lung cancer model [34]. Short-term exposure to bortezomib produces opposing effects resulting in inhibition of tumor cell growth [34], similar to the anti-tumorigenic properties of 10 day TQ treatment [21]. It is not known whether the deleterious effects of long-term TQ and bortezomib, both relatively non-specific inhibitors of NF- $\kappa B$ with additional cellular effects [4-6, 34], are due to their actions on NF- $\mathrm{kB}$ activity in these preclinical models. However, because of the interest in using systemic NF- $\kappa B$ inhibitors as mono-therapy or in combination with other chemotherapeutic drugs in clinical trials in ovarian cancer patients [35], possible side effects that limit drug efficacy, promote toxicity or have frankly deleterious effects are highly relevant clinically.

In conclusion, our results emphasize the strong potential of targeting DNA damage as a therapeutic approach to improve cisplatin response in ovarian cancer. However, the choice of drug to combine with cisplatin is critical, since we have shown that $\mathrm{TQ}$, a very promising therapeutic agent in preclinical cancer models, had an unexpected deleterious 
effect through promotion of ascites formation in a syngeneic model. Since TQ is a potential candidate for future clinical trials in cancer patients [5-7], this finding provides caution for its future use in patients with ovarian cancer.

\section{Additional file}

Additional file 1: Figure S1. Isobologram analysis of combination effects of TQ and cisplatin in human ovarian cancer cells in vitro.

\section{Abbreviations}

IL: Interleukin; NF-kB: Nuclear factor-kappaB; NGL: NF-kB-GFP-Luciferase; TQ: Thymoquinone.

\section{Competing interests}

The authors declare that they have no competing interests.

\begin{abstract}
Authors' contributions
AJW oversaw the experiments, performed tumor cell injections into C57BL/6 mice, performed dissections and collection of ascites/peritoneal lavages, performed in vitro drug treatment experiments and western blots, analyzed the data, and drafted the manuscript. JS was responsible for mouse husbandry, maintained cell lines, processed tumor tissue for paraffin-embedding, processed ascites/peritoneal lavage fluid for RNA and protein extraction and preparation of cytospin slides, performed immunofluorescence staining in tumor and ascites samples, and prepared RNA for CDNA synthesis and QPCR analysis. WB was responsible for mouse husbandry, assisted in mice dissections and ascites processing, performed QPCR analysis, performed cytospin counts, and assisted in preparation of the manuscript. FY conceived the study, provided the C57BL/6 mice, consulted on experimental design and data analysis and shared final editorial oversight of the manuscript. DK conceived the study, consulted on experimental design and data analysis, and shared final editorial oversight of the
\end{abstract} manuscript. All of the authors have read and approved the final version.

\section{Acknowledgments}

We acknowledge The Vanderbilt Imaging Core, and The Allergy/Pulmonary \& Critical Care Med Division Immunohistochemistry Core.

\section{Grant support}

DOD W81XWH-11-1-0509 (AJW); DOD W81XWH-09-1-0459, NIH

R01CA113734 (FY); NIH K08CA148887 (DK).

\section{Author details \\ 'Department of Obstetrics and Gynecology, Division of Gynecologic Oncology, Vanderbilt University School of Medicine, Nashville, TN, USA. ${ }^{2}$ Department of Cancer Biology, Vanderbilt University Medical Center, Nashville, TN, USA. ${ }^{3}$ Vanderbilt-Ingram Cancer Center, Vanderbilt University Medical Center, Nashville, TN, USA. ${ }^{4}$ Department of Obstetrics and Gynecology, Vanderbilt University Medical Center, B1100 Medical Center North, Nashville, TN 37232, USA.}

\section{Received: 24 April 2015 Accepted: 8 July 2015}

Published online: 28 July 2015

\section{References}

1. Siegel RL, Miller KD, Jemal A. Cancer statistics, 2015. CA Cancer J Clin. 2015;65(1):5-29. doi:10.3322/caac.21254.

2. Bast Jr RC, Hennessy B, Mills GB. The biology of ovarian cancer: new opportunities for translation. Nat Rev Cancer. 2009;9(6):415-28. doi:10.1038/nrc2644.

3. Muggia F. Platinum compounds 30 years after the introduction of cisplatin: implications for the treatment of ovarian cancer. Gynecol Oncol. 2009;112(1):275-81. doi:10.1016/j.ygyno.2008.09.034.

4. Sethi G, Ahn KS, Aggarwal BB. Targeting nuclear factor-kappa B activation pathway by thymoquinone: role in suppression of antiapoptotic gene products and enhancement of apoptosis. Mol Cancer Res. 2008;6(6):1059-70. doi:10.1158/1541-7786.MCR-07-2088.
5. Ahmad A, Husain A, Mujeeb M, Khan SA, Najmi AK, Siddique NA, et al. A review on therapeutic potential of Nigella sativa: a miracle herb. Asian Pac J Trop Biomed. 2013;3(5):337-52. doi:10.1016/S2221-1691(13)60075-1.

6. Rahmani AH, Alzohairy MA, Khan MA, Aly SM. Therapeutic implications of black seed and its constituent thymoquinone in the prevention of cancer through inactivation and activation of molecular pathways. Evid Based Complement Alternat Med. 2014;2014:724658. doi:10.1155/2014/724658.

7. Abukhader MM. Thymoquinone in the clinical treatment of cancer: fact or fiction? Pharmacogn Rev. 2013;7(14):117-20. doi:10.4103/0973-7847.120509.

8. Hernandez L, Hsu SC, Davidson B, Birrer MJ, Kohn EC, Annunziata CM. Activation of NF-kappaB signaling by inhibitor of NF-kappaB kinase beta increases aggressiveness of ovarian cancer. Cancer Res. 2010;70(10):4005-14. 10.1158/0008-5472.CAN-09-3912.

9. Meylan E, Dooley AL, Feldser DM, Shen L, Turk E, Ouyang C, et al. Requirement for NF-kappaB signalling in a mouse model of lung adenocarcinoma. Nature. 2009;462(7269):104-7. doi:10.1038/nature08462.

10. Karin M. The IkappaB kinase-a bridge between inflammation and cancer. Cell Res. 2008;18(3):334-42. doi:10.1038/cr.2008.30.

11. Balkwill F, Coussens LM. Cancer: an inflammatory link. Nature. 2004;431 (7007):405-6. doi:10.1038/431405a.

12. Aggarwal BB. Nuclear factor-kappaB: the enemy within. Cancer Cell. 2004;6(3):203-8. doi:10.1016/j.ccr.2004.09.003.

13. Coussens LM, Werb Z. Inflammation and cancer. Nature. 2002;420(6917):860-7. doi:10.1038/nature01322.

14. Al-Amri AM, Bamosa AO. Phase I safety and clinical activity study of thymoquinone in patients with advanced refractory malignant disease. Shiraz E-Med J. 2009;10(3):107-11.

15. Galluzzi L, Senovilla L, Vitale I, Michels J, Martins I, Kepp O, et al. Molecular mechanisms of cisplatin resistance. Oncogene. 2012;31(15):1869-83. doi:10.1038/onc.2011.384.

16. Miow QH, Tan TZ, Ye J, Lau JA, Yokomizo T, Thiery JP, et al. Epithelial-mesenchymal status renders differential responses to cisplatin in ovarian cancer. Oncogene. 2014. doi:10.1038/onc.2014.136.

17. Gilmore TD, Herscovitch M. Inhibitors of NF-kappaB signaling: 785 and counting. Oncogene. 2006;25(51):6887-99. doi:10.1038/sj.onc.1209982.

18. DelloRusso C, Welcsh PL, Wang W, Garcia RL, King MC, Swisher EM. Functional characterization of a novel BRCA1-null ovarian cancer cell line in response to ionizing radiation. Mol Cancer Res. 2007:5(1):35-45. doi:10.1158/1541-7786.MCR-06-0234.

19. Wilson AJ, Holson E, Wagner F, Zhang YL, Fass DM, Haggarty SJ, et al. The DNA damage mark pH2AX differentiates the cytotoxic effects of small molecule HDAC inhibitors in ovarian cancer cells. Cancer Biol Ther. 2011;12(6):484-93. doi:10.4161/cbt.12.6.15956.

20. Wilson AJ, Lalani AS, Wass E, Saskowski J, Khabele D. Romidepsin (FK228) combined with cisplatin stimulates DNA damage-induced cell death in ovarian cancer. Gynecol Oncol. 2012;127(3):579-86. doi:10.1016/j.ygyno.2012.09.016

21. Wilson AJ, Barham W, Saskowski J, Tikhomirov O, Chen L, Lee HJ, et al. Tracking NF-kappaB activity in tumor cells during ovarian cancer progression in a syngeneic mouse model. J Ovarian Res. 2013;6(1):63. doi:10.1186/1757-2215-6-63.

22. Wilson AJ, Liu AY, Roland J, Adebayo OB, Fletcher SA, Slaughter JC, et al. TR3 modulates platinum resistance in ovarian cancer. Cancer Res. 2013;73(15):4758-69. doi:10.1158/0008-5472.CAN-12-4560.

23. Chou TC, Talalay P. Quantitative analysis of dose-effect relationships: the combined effects of multiple drugs or enzyme inhibitors. Adv Enzym Regul. 1984;22:27-55.

24. Livak KJ, Schmittgen TD. Analysis of relative gene expression data using real-time quantitative PCR and the 2(-Delta Delta C(T)) Method. Methods. 2001;25(4):402-8. doi:10.1006/meth.2001.1262.

25. Everhart MB, Han W, Sherrill TP, Arutiunov M, Polosukhin W, Burke JR, et al. Duration and intensity of NF-kappaB activity determine the severity of endotoxin-induced acute lung injury. J Immunol. 2006;176(8):4995-5005.

26. Stathopoulos GT, Sherrill TP, Han W, Sadikot RT, Yull FE, Blackwell TS, et al. Host nuclear factor-kappaB activation potentiates lung cancer metastasis. Mol Cancer Res. 2008;6(3):364-71. doi:10.1158/1541-7786.MCR-07-0309.

27. Khabele D, Fadare O, Liu AY, Wilson AJ, Wass E, Osteen K, et al. An orthotopic model of platinum-sensitive high grade serous fallopian tube carcinoma. Int J Clin Exp Pathol. 2012;5(1):37-45.

28. Khabele D, Son DS, Parl AK, Goldberg GL, Augenlicht LH, Mariadason JM, et al. Drug-induced inactivation or gene silencing of class I histone 
deacetylases suppresses ovarian cancer cell growth: implications for therapy. Cancer Biol Ther. 2007;6(5):795-801.

29. Wilson AJ, Byun DS, Nasser S, Murray LB, Ayyanar K, Arango D, et al. HDAC4 promotes growth of colon cancer cells via repression of p21. Mol Biol Cell. 2008;19(10):4062-75. doi:10.1091/mbc.E08-02-0139.

30. Connelly L, Barham W, Onishko HM, Sherrill T, Chodosh LA, Blackwell TS, et al. Inhibition of NF-kappa B activity in mammary epithelium increases tumor latency and decreases tumor burden. Oncogene.

2011;30(12):1402-12. doi:10.1038/onc.2010.521.

31. Roepke M, Diestel A, Bajbouj K, Walluscheck D, Schonfeld P, Roessner A, et al. Lack of p53 augments thymoquinone-induced apoptosis and caspase activation in human osteosarcoma cells. Cancer Biol Ther. 2007;6(2):160-9.

32. Gurung RL, Lim SN, Khaw AK, Soon JF, Shenoy K, Mohamed Ali S, et al. Thymoquinone induces telomere shortening, DNA damage and apoptosis in human glioblastoma cells. PLoS One. 2010;5(8), e12124. doi:10.1371/journal.pone.0012124.

33. Banath JP, Klokov D, MacPhail SH, Banuelos CA, Olive PL. Residual gammaH2AX foci as an indication of lethal DNA lesions. BMC Cancer. 2010;10:4. doi:10.1186/1471-2407-10-4

34. Karabela SP, Psallidas I, Sherrill TP, Kairi CA, Zaynagetdinov R, Cheng DS, et al. Opposing effects of bortezomib-induced nuclear factor-kappaB inhibition on chemical lung carcinogenesis. Carcinogenesis. 2012;33(4):859-67. doi:10.1093/carcin/bgs024.

35. Aghajanian C, Blessing JA, Darcy KM, Reid G, DeGeest K, Rubin SC, et al. A phase II evaluation of bortezomib in the treatment of recurrent platinum-sensitive ovarian or primary peritoneal cancer: a Gynecologic Oncology Group study. Gynecol Oncol. 2009;115(2):215-20. doi:10.1016/.ygyno.2009.07.023

\section{Submit your next manuscript to BioMed Central and take full advantage of:}

- Convenient online submission

- Thorough peer review

- No space constraints or color figure charges

- Immediate publication on acceptance

- Inclusion in PubMed, CAS, Scopus and Google Scholar

- Research which is freely available for redistribution 